\title{
Implementation of a System for Localization and Positioning of Vehicles using GPS and GPRS Technology
}

\author{
Shreenivas Jog, M. S. Sutaone, Bhushan Joshi, Vrushali Bahirat, Jinesh Nair, and Astitva Karunesh
}

\begin{abstract}
The objective of this paper is to describe a system, implemented using GPS and GSM technologies, whereby a command is sent to the system in the form of a SMS by system registered Cell phone and the system responds to it by transmitting its current coordinates in the form of Latitude and Longitude using a reply SMS to same Cell phone. The system gives current vehicle location whenever needed with reliable accuracy. The system uses GSM/GPRS, and GPS Technology, helping in efficient monitoring of the desired vehicles. The paper also discusses the proposed GPS based vehicle Tracking System using GPRS technology in which the coordinates are forwarded to data server at designated Static IP address. The position of the vehicle can be traced on Google / Local maps. The paper gives functional, Technical description and Software implementation for the GPS and GSM/ GPRS based Vehicle Tracking System.
\end{abstract}

Index Terms-GPS ProGin SR-87, GSM / GPRS SIM300, localization of vehicles, microcontroller 89S52, static IP.

\section{INTRODUCTION}

The paper describes the idea of tracking a vehicle using latest technology of GPS and GSM. The number of industry related vehicles like oil tankers (trucks), vans carrying huge amount of money for ATMs are increasing at a very fast rate and keeping a track of these vehicles is becoming difficult day by day. To keep a check on these kinds of vehicles such technologies prove to be very useful since in case of theft or missing of these vehicles, they can be easily traced on the website or cell-phones. Mobile technologies such as GSM / GPRS and GPS can be used for displaying the current position of the vehicle indicating the latitude, longitude and height from sea level. [1] This displaying of location of the vehicle can be done by a number of methods. The location can be sent via SMS to a GSM modem kept at the control station or to a cell phone. It can be indicated on LCD at the control station. The coordinates of the vehicle can also be transmitted to a static IP address by using GPRS Technology, thus linking it to Local/Google Maps. GPRS enables the introduction of packet data capabilities into the existing PCS and cellular networks using the TDMA or GSM Technology. [2]

Using GPS one's exact location (longitude, latitude and

Manuscript received December 29, 2012; revised April 26, 2013.

Shreenivas Jog and M. S. Sutaone are with E and TC Department, College of Engineering, Pune, India (e-mail: srjog_etc@rediffmail.com, mssutaone@gmail.com)

Bhushan Joshi is with IUCAA, India (e-mail: bhushan@iucaa.ernet.in)

Vrushali Bahirat, Jinesh Nair, and Astitva Karunesh are with D. Y. Patil Institute of Engineering and Technology, Pimpri, Pune, India (e-mail: vrushalibahirat@gmail.com, jineshnair10@gmail.com, astitva.karunesh@ gmail.com) height from sea level) can be obtained within a range of 20 meters. The precise time (Universal Time Coordinated (UTC)) can be obtained within a range of $60 \mathrm{~ns}$ to $5 \mathrm{~ns}$. Speed and direction of travel (course) can be derived from these co-ordinates as well as the time. The distance to the satellite (Pseudo range) can be determined by using the known transit time.

\section{FUNCTIONAL DESCRIPTION}

The SMS Based Vehicle Tracking System has been implemented using GPS ProGin SR-87 and GSM / GPRS module SIM300. Fig. 1 shows the block diagram for the implementation of a GPS based vehicle tracking and monitoring system. GPS module receives signals from a series of satellites, and calculates its current geographical location, speed etc. The microcontroller processes this data through MAX232 level converter. GSM module receives request for sending vehicle location from system registered cell phone. The GSM module sends reply SMS to same cell phone giving the vehicle location.

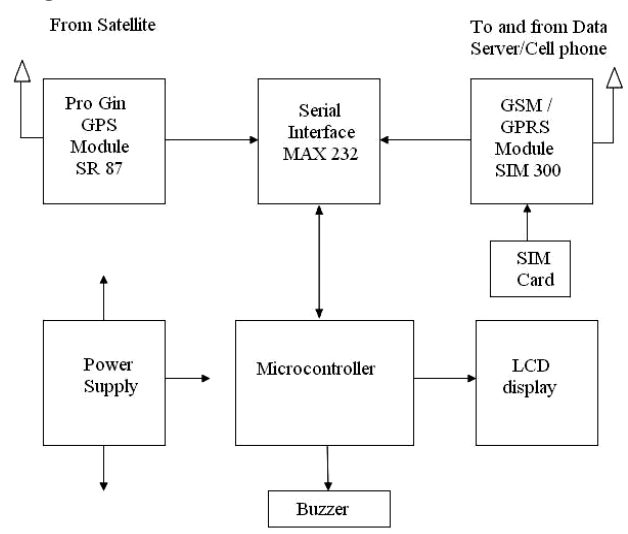

Fig. 1. Block diagram for GPS and GSM/GPRS based system.

The information about vehicle location i.e. latitude, longitude and height from sea level can also be stored for later retrieval or frequently transmitted to a control station where it can be displayed on a high-resolution geographical map or can be directly sent to a data server with designated Static IP address. The LCD at the system end indicates coordinates and speed of the vehicle. The system consists of following modules.

\section{A. Progin SR-87 GPS Module}

The ProGin SR-87 series GPS module incorporates high sensitivity, low power consumption and SiRF chipset solution in a compact design. The module tracks up to 20 satellites at a time while offering fast time-to-first-fix and 1Hz navigation update. [3] The SR-87 design utilizes the 
latest surface mount technology and high level circuit integration to achieve superior performance while minimizing dimensions $(25.4 \times 25.4 \times 7 \mathrm{~mm} 3)$ and power consumption. This hardware capability combined with software intelligence makes the board easy to be integrated and used in all kinds of navigation applications or products. This module is basically functional in decoding the data sent by the satellites into GPS frames which contain the position, speed etc. of the device. The co-ordinates take some time before they become clearly visible if viewed, due to the time taken for decoding of the frames. This unit is very suitable for broad applications such as handheld PDA, PPC or other battery operated navigation systems. [3]

\section{B. The NMEA-0183 Data Interface}

This interface is used by the system in order to relay computed GPS variables such as position, velocity, course etc. to a peripheral (e.g. computer screen or transceiver). The most important elements of receiver information are broadcast via NMEA-0183 data interface in a special data format. This format is standardized by the National Marine Electronics Association (NMEA) to ensure that data exchange takes place without any problems.

\section{MAX 232}

MAX232 is an integrated circuit that converts signals from an RS232 serial port to signals suitable for use in TTL compatible digital logic circuits. MAX232 is a dual driver/receiver which typically converts the RX, TX, CTS and RTS signals. [4] Here, in this device, the MAX 232 is expected to serially interface the GPS module with the microcontroller so that the microcontroller can accept the GPS frames sent by the GPS module in an efficient way.

\section{Microcontroller 89 S52}

In this system, the microcontroller $89 \mathrm{~S} 52$ plays the most vital role. [5] The code burnt in the microcontroller decodes the data received from the satellite using the concept of a counter, thus converting the GPS frames received from the GPS module in an understandable format. Moreover, the microcontroller is also responsible to send the required information through MAX232 and GPRS/GSM to the desired static IP address, for which socket programming is required. $89 \mathrm{~S} 52$ is sufficient to handle the memory requirements for the GSM approach while more efficient microcontroller series is required for the implementation of the Static IP approach. We propose microcontroller LPC2148 for GPRS based Vehicle Tracking System [6]

\section{E. GSM/GPRS Module SIM 300}

GSM/GPRS module SIM 300 is a low power consumption device. It supports TCL band GSM/GPRS 900/1800/1900 $\mathrm{MHz}$. The general features also include a GPRS multi-slot class 10 and a GPRS mobile station Class B. The dimensions of the device are $33 \mathrm{~mm} \times 33 \mathrm{~mm} \times 3 \mathrm{~mm}$ and weighs $7.8 \mathrm{gm}$. It is controlled via AT commands. It has a SIM application toolkit. Supply voltage range supported is $3.4 \mathrm{~V}$ to $4.5 \mathrm{~V}$. It also includes PPP stack. Non transparent mode is incorporated in the modem. [7] Along with the coordinates the speed, temperature etc. of the vehicle can also be transmitted once the device is in dedicated mode (i.e., a TCH is assigned to it). [7]

\section{F. Website (Static IP Address)}

A static IP address is a number (in the form of a dotted quad) that is assigned to a computer by an Internet Service provider (ISP) to be its permanent address on the Internet. A System with a static IP address can be accessed from anywhere in the world. [8] The required fields of information are pushed by GPRS to this particular static IP address. All this information can be displayed on the map as well as website. The information can be stored for later retrieval or can be transmitted frequently for simultaneous access. A track of system movement can be kept by analyzing the route followed for a particular time interval and average speed of the vehicle.

\section{SOFTWARE IMPLEMENTATION}

The steps for software implementation of the GSM/GPRS based system are discussed below. Fig. 2 and Fig. 3 show Flowcharts for Software Implementation.

\section{A. Algorithm for GPS, GSM / GPRS Based System}

1) Start.

2) Separate out the latitude and longitude from the \$GPRMC frame.

3) Select the method of transmitting coordinates whether GSM (using SMS) or GPRS (to the static IP).

4) If GPRS is used then establish connection with the remote server having static IP.

5) Send data packets to the server.

6) Terminate the connection.

7) If data is to be displayed using a GSM, then send the SMS containing the position information to the cell phone.

\section{B. Algorithm at the Data Server / User End}

1) Start.

2) Check if data is received.

3) Acquire the data.

4) Send data to the database using VB.net.

5) Acquire data from database.

6) Plot the data on the map using ASP.net and PHP.net.

7) End.

\section{Flowchart at the System End}

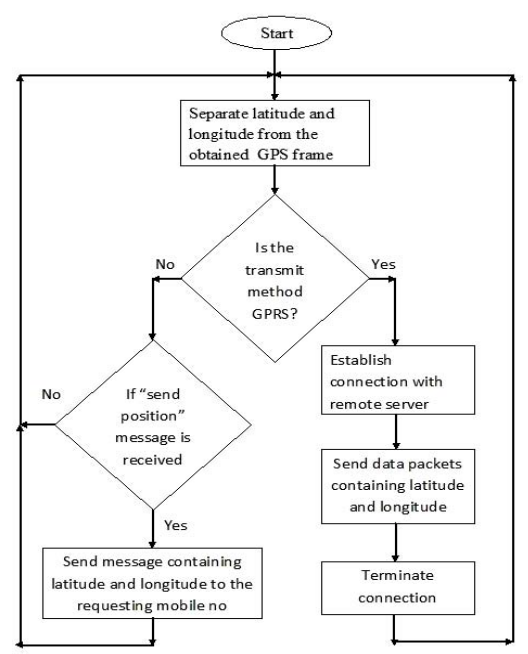

Fig. 2. Flowchart for software implementation at the system end. 


\section{Flowchart at the Data Server / User End}

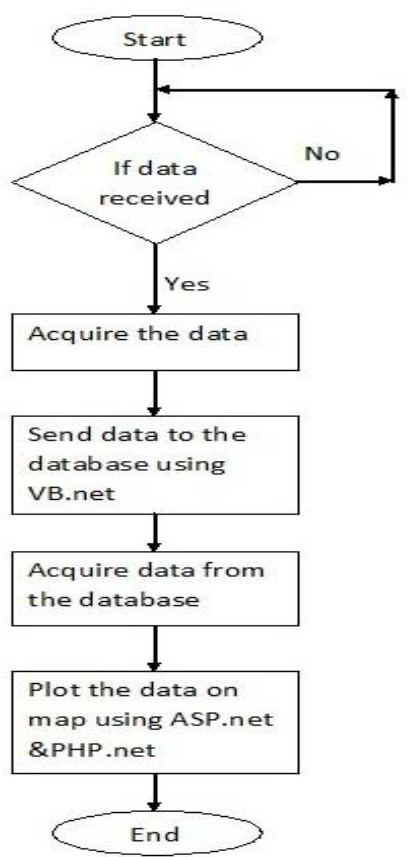

Fig. 3. Flowchart for software implementation at data server/user end.

\section{RESUlTS FOR THE IMPLEMENTED SYSTEM}

Fig. 4 and Fig. 5 indicate the Hardware and LCD for SMS based Vehicle Tracking System implemented using GPS and GSM. Figure 6 shows the different NMEA [3] data formats in which the frames are obtained from GPS by establishing a connection of the system with Hyper Terminal. A number of frame formats are obtained from GPS module ProGin SR-87 like \$GPGSA, \$GPGSV, \$GPVTG, \$GPRMC etc. The main frame of interest is \$GPRMC which contains the system information like position (latitude, longitude, altitude), speed, time etc. which is decoded by the microcontroller 89S52, thus giving the data in the desired format.

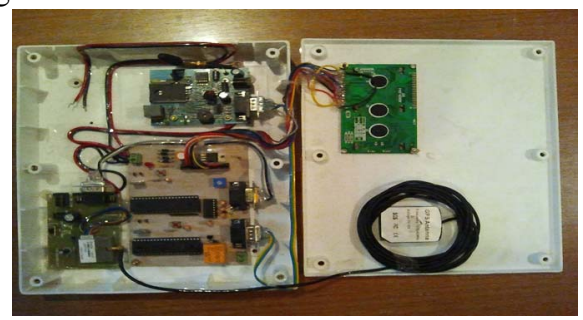

Fig. 4. GPS and GSM based vehicle tracking system.

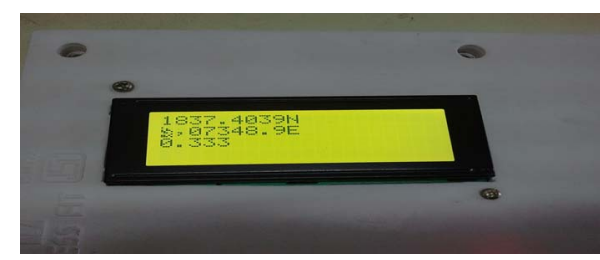

Fig. 5. Position and speed as indicated on LCD of the system.

The Fig. 7 shows the data observed on GSM module SIM300 for receiving request for position by SMS from system registered cell phone and reply by sending current location through SMS to same cell phone.

Currently the system is implemented using GSM technology whereby a command is sent to it in the form of SMS by system registered cell phone and the system responds to it by transmitting its current coordinates in the form of latitude and longitude using an a reply SMS to same cell phone. Figure 8 shows the command sent and the reply SMS obtained from the system on registered cell phone.

GPRS technology can be used for tracing the system on a website whereby the coordinates are sent on a static IP address thus displaying the system's position on Google/local maps. The proposed GPRS based Vehicle Tracking System is intended to use microcontroller LPC2148 along with same GPS and GSM module used for SMS based system.

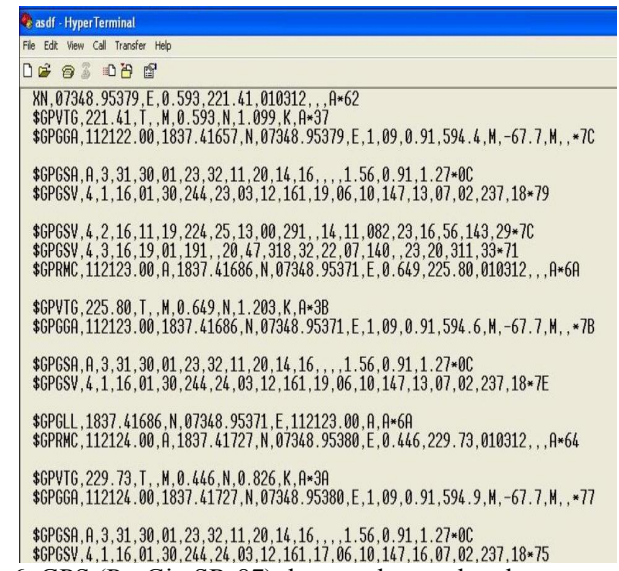

Fig. 6. GPS (ProGin SR-87) data as observed on hyper terminal.

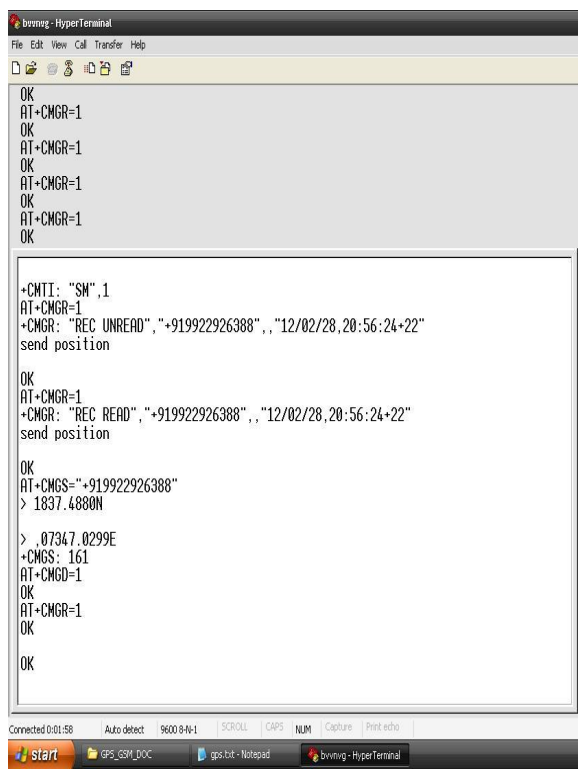

Fig. 7. GSM (SIM 300) data as observed on hyper terminal.

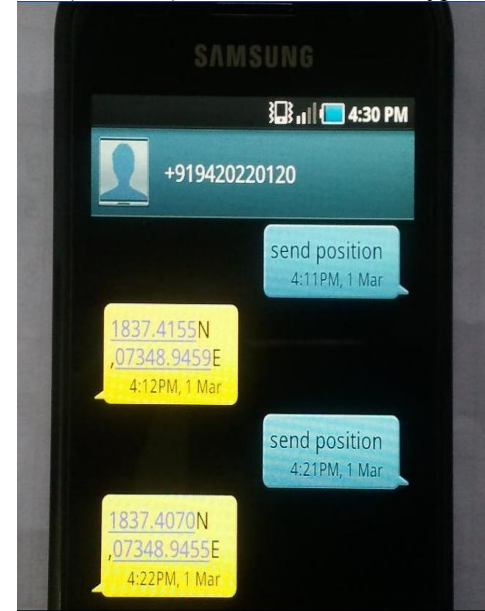

Fig. 8. Command sent to obtain coordinates and latitude, longitude obtained on cell phone. 


\section{FUTURE SCOPE}

The system performance can improve with the use of technologies like EDGE (provides modulation improvement over GPRS) which gives much more data rate as compared to GPRS. In principle, all network elements and events should be monitored for possible failures.

Along with basic positioning, additional parameters of the vehicle like temperature, engine parameters, RPM, speed analysis, fuel level and door status etc can be transmitted using GSM/GPRS technology to the registered cell phone or static IP. [9]

The system accuracy and speed of data transmission can be improved further by using differential GPS (DGPS) and mobile Wi-MAX technologies. The features like live video transmission of system location can be added in basic configuration of system. [9]

The System performance can improve by testing the system for Environmental, Dynamic and EMI /EMC tests. Ruggedised GPS and GSM /GPRS based Vehicle Tracking System can be used for stringent environment like Military based applications or tough automotive applications. [10]

\section{CONCLUSION}

The SMS based system using GPS and GSM modules has been implemented and tested. This system is a low cost solution to the currently existing expensive systems with similar applications. It gives acceptable accuracy for localized vehicle tracking. System size is small thus making it simple to mount into vehicles. In the GSM based system users can easily trace the system without the requirement of Laptop/ Computers and Internet access technologies. The system is also capable of giving localized coordinate display along with speed on a LCD, in turn helping the user for navigation. The system can be modified for GPRS based applications to send coordinates and speed to static IP address.

\section{ACKNOWLEDGMENT}

The authors would like to sincerely thank Sayed Faizan, Swapnil Karne, Shahid Akhtar from Pad. Dr. D.Y.Patil Institute of Engineering Technology for their valuable help for this project work.

\section{REFERENCES}

[1] J. Bell, Basic GPS Navigation, the practical guide to GPS navigation, 2008.

[2] L. Harte, B. Bromley, and M. Devis, Introduction to GSM, $2^{\text {nd }}$ edition, 2009.

[3] Datasheet. Specifications and features. [Online]. Available: http://www.progin.com.tw/sr87_en.htm.

[4] Datasheet and specifications $/$ features of MAX 232. [Online]. Available:

http://www.datasheetcatalog.org/datasheet/texasinstruments/max232.p df.

[5] Datasheet and specifications of 89S52 microcontroller. [Online]. Available:

http://www.atmel.com/PFResults.aspx\#(data:(category:'34864',type: !( 16)),sc:3).

[6] Datasheet and specifications of LPC 2148 microcontroller. [Online]. Available: http://www.keil.com/dd/chip/3880.htm.
[7] Datasheet of GSM/GPRS module SIM 300. [Online]. Available: http://www.alldatasheet.com/view.jsp?Searchword=SIM300.

[8] A. S. Tanenbaum, Computer Network, Prentice Hall, $4^{\text {th }}$ edition, 2002.

[9] S. Jog, M. S. Sutaone, and V. V. Badawe, "Performance Improvement of GPS Based Vehicle Tracking System using DGPS and Mobile Wi-Max," International Journal on Computer Science Engineering and Technology (IJCSET), vol. 1, no. 8, Sept. 2011, pp. 491-495.

[10] S. Jog, M. S. Sutaone, and V. Badave, "Ruggedisation Methodologies for GPS based Vehicle Tracking System," in Proc. of IEEE (IACSIT) International Conference on Electronic Computer Technology (ICECT-2011), vol. 2, pp. 214-218, Kanyakumari, India, April-2011.

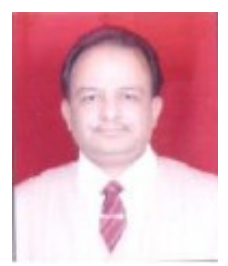

Shreenivas Jog is presently persuing for Ph.D. in E and TC at College of Engineering Pune, affiliated to University of Pune, India. He has completed B.E and M.E (E and TC) from College of Engineering Pune. He has total experience of 23 years in academics / Industry (R \& D). He has also worked as Dy. Manager (R\&D) MELTRON, Pune, India. His area of interest includes GPS, Mobile and Wireless Communication, EMI/EMC,Ruggedised, Defence Communications systems. He has published 46 papers in National and International Conferences/Journals. He is presently working as Professor and HOD (E and TC) in Pd. Dr. D.Y. Patil Institute of Engineering and Technology, Pimpri, Pune, India. He is Fellow of IETE and Member of IEEE, IE (I) and ISTE. He is a Chairman of IETE Pune, India and Ex-Officio Governing Council Member of IETE at National level. He is also member of TPC and Publications Committee of IETE India.

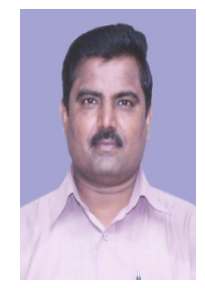

M. S. Sutaone is working as a Dean and Professor E and TC department, College of Engineering Pune and he has 30 years experience in academics and he has published more than 60 papers in National/ International Conferences / Journals. His area of interest includes DSP, Image Processing, Mobile, VLSI and Wireless Systems.

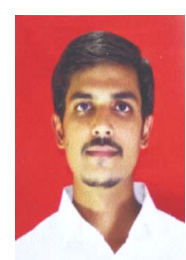

Bhushan Joshi has completed B.E. (Electronics) from university of Pune. His area of interest is embedded system design and instrumentation. $\mathrm{He}$ has 4 years experience in embedded industry. He is currently working as an Electronics Engineer in IUCAA (Inter University Centre for Astronomy and Astrophysics).

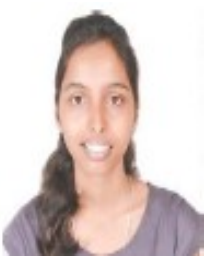

Vrushali Bahirat has completed B.E (Electronics and Telecommunication) from, Pd. Dr. D.Y. Patil Institute of Engineering and Technology, Pimpri, Pune. She has published 2 papers in National and International Conferences. She is a student member of IETE.

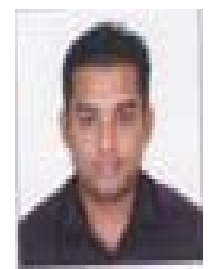

Jinesh Nair has completed Graduation in Engineering in the field of Electronics and Telecommunications from Padmashree Dr. D. Y. Patil Institute of Engineering and Technology, Pimpri, Pune in the year 2011-12. He is a student member of the IETE. He has also won a prize in national level technical competition.

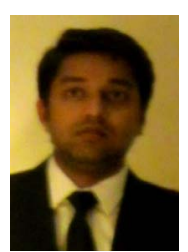

Astitva Karunesh has completed his Graduation in Engineering in the field of Electronics and Telecommunications from Padmashree Dr. D. Y. Patil Institute of Engineering and Technology, Pimpri, Pune in the year 2011-12.He is a student member of the IETE. $\mathrm{He}$ has also won prize in National level Technical competition. 